\title{
Stroke in Senegalese Young Women: About 109 Cases
}

\section{Arame Gaye*, Ousmane Cissé, Salaheddine Mourabit, Marième Soda Diop Sène, Ngor Side Diagne, Moustapha Ndiaye, Amadou Gallo Diop}

Neurology Department of Fann National Teaching Hospital, University of Dakar, Dakar, Senegal

\section{Email address:}

gayarame45@gmail.com (A. Gaye)

${ }^{*}$ Corresponding author

\section{To cite this article:}

Arame Gaye, Ousmane Cissé, Salaheddine Mourabit, Marième Soda Diop Sène, Ngor Side Diagne, Moustapha Ndiaye, Amadou Gallo Diop. Stroke in Senegalese Young Women: About 109 Cases. Science Journal of Clinical Medicine. Vol. 7, No. 1, 2018, pp. 1-8. doi: 10.11648/j.sjcm.20180701.11

Received: December 15, 2017; Accepted: January 25, 2018; Published: March 5, 2018

\begin{abstract}
Considered for a long time as pathology of the elderly, stroke is more and more frequent in the young subject, 12 to $15 \%$ of the stroke occurs in the people under 50 years old. The situation is alarming because the consequences are tragic on the economic and social levels. We will be interested in the stroke of the young women. This is a cross-sectional descriptive study over 22 month in neurology department in Fann teaching hospital, period from August 2012 to June 2014. This allowed to the compilation of 109 patients with stroke according to the WHO definition and aged between 16 and 50 years. This study focuses on 109 women aged between 17 and 50 years with an average age of 39.95 years. The most representative age range is between 36 and 45 years. Only $3.7 \%$ of patients had a level of education higher than baccalaureate, 59.6\% were not in school.57.8\% had an ischemic stroke and $42.2 \%$ had a hemorrhagic stroke, with an average age of 39 years for ischemic stroke and 40 years for hemorrhagic stroke. The most important cardiovascular risk factors for ischemic stroke were dyslipidemia $(73 \%)$, high blood pressure $(42.9 \%)$, sedentary lifestyle $(38.1 \%)$, and estrogen-progestin contraception intake were found in $23.8 \%$ of cases. According to the TOAST classification, the etiology is embolic heart disease (25.4\%), macroangiopathy $(17.5 \%)$, microangiopathy $(7.9 \%)$, others $(15.9 \%)$, and in $41.2 \%$ of the patients the etiology is not found. The main cardiovascular risk factors in hemorrhagic stroke were hypertension (80.4\%), dyslipidemia (58.7\%), sedentarily (59.1\%), migraine $(23.9 \%)$ an oestroprogestative contraception intake $(6.5 \%)$. The etiologies of hemorrhagic stroke are arterial hypertension $(80.4 \%)$, left middle cerebral artery aneurysm $(6.5 \%)$, arteriovenous malformation $(4.3 \%)$, infectious arteritis $(4.3 \%)$, hemostasis disorder $(2.2 \%)$, and undetermined origin $(2.2 \%)$. Bad eating habits, sedentary lifestyle lead an increase cardiovascular risk factors and, at the same time, the stroke rate in young women. Prevention must go through a good awareness-raising policy.
\end{abstract}

Keywords: Cardiovascular Risk Factors, Stroke, Young Women

\section{Introduction}

Stroke is defined as the rapid development of localized or global clinical signs of brain dysfunction with symptoms lasting more than 24 hours leading to death, without any other apparent cause than a vascular origin. (World Health Organization)

Considered beforehand as pathology of the elderly, stroke is increasingly found in developing countries, in the young subject and particularly in women.

However, this situation is deplorable in view of the heavy professional, social and economic consequences for this person whose income is most often the main source of expenditure for that mother who must have all her physical and psychic abilities in order to care of her home and other...

10 million people in the world are affected by stroke, a quarter of those are under 65 years of age.

12 to $15 \%$ of stroke occurs in people under 50 years of age $[12,24]$. The incidence rate varies between 10 and 23 cases per 100000 inhabitants in the word [24].

According to data from the 2014 Behavioral Risk Factor 
Surveillance System / Centers of Disease Control and Prevention [BRFSS (CDC)], $2.8 \%$ of men and $2.8 \%$ of women $\geq 18$ years of age had a history of stroke; $2.5 \%$ of non-Hispanic whites, $4.5 \%$ of non-Hispanic blacks, $1.8 \%$ of Asian/Pacific Islanders, 2.4\% of Hispanics (of any race), $5.4 \%$ of American Indian/Alaska Natives, and $4.7 \%$ of other races or multiracial people had a history of stroke [29]. These rates are not negligible, given the serious consequences.

Stroke is the first cause of acquired adult disability, the second leading cause of dementia, the third leading cause of mortality (after cancer and myocardial infarction) and the major cause of depression worldwide, whence the importance of knowing the etiologies and the risk factors in order to prevent it.

Therefore in this study, among these young women, the cardiovascular risk factors are predominated by dyslipidemia and high blood pressure. On the other hand, the rate of stroke related to oestrogen-progestin contraception intake is really low.

\section{Method}

Our study framework was the Neurology department of Fann National Teaching Hospital.

Type of study:

This is a cross-sectional descriptive study over 22 month in neurology department of Fann teaching hospital, period from August 2012 to June 2014. This allowed to the compilation of 109 patients with stroke according to the WHO definition and aged between 16 and 50 years.

Objectives of this study:

To determine the risk factors associated with stroke in young women

To determine the clinical, paraclinical and evolutionary profile of the young woman's stroke.

The data collection was done through fact sheets comprising 6 parts:

1. Biographical data, 2. anamnesis, 3. physical examination, 4. paraclinical assessment, 5. treatment received, 6. evolution

The treatment of data collected in our patients was carried out by the statistical software SPSS version 17.0; and we used the sphinx version 4.5 software to make the input model.

\section{Results}

\subsection{Epidemiological Study}

\subsubsection{Age and Number of Patients}

Our study included a sample of 109 patients, aged between 17 and 50 years, with an average age of 39.95 years. The most significant age class is between 36 and 45 years. Figure 1.

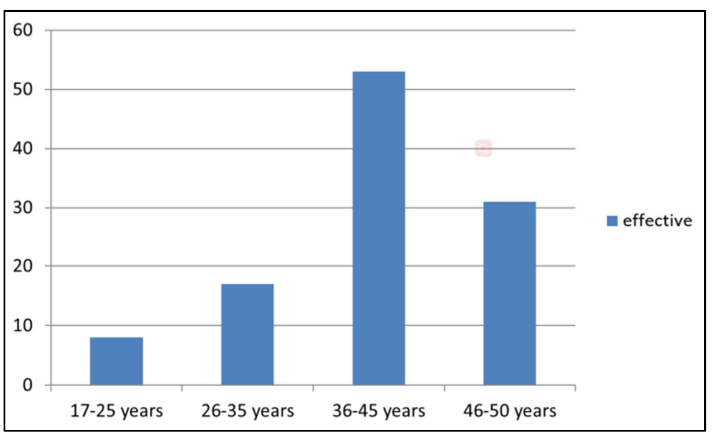

Figure 1. Number of patients by age class.

\subsubsection{Level of Instruction}

Most of the patients were not in school. Only $40.4 \%$ of the patients are educated, which $3.7 \%$ are at a higher level (after baccalaureate). Cf. Table 1

Table 1. Level of instruction.

\begin{tabular}{lll}
\hline Level of instruction: & Effective & Percentage \% \\
\hline koranic school & 1 & 0.9 \\
Primary school & 26 & 23.9 \\
Secondary school & 13 & 11.9 \\
After baccalaureate & 4 & 3.7 \\
Unschooled & 65 & 59.6 \\
Overall & 109 & 100 \\
\hline
\end{tabular}

\subsubsection{Marital Status}

Most of our patients (67.9\%) are married. Figure 2

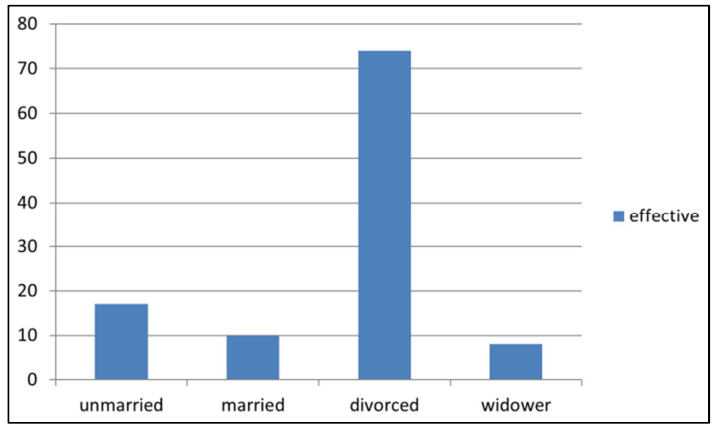

Figure 2. Marital status

\subsubsection{Geographic Origin}

The majority of our patients are from Senegal, there are one Mauritanian and one Gambian. Table 2

Table 2. Geographic origin

\begin{tabular}{lll}
\hline Localities & Effective & Percentage \% \\
\hline Dakar & 83 & 76,1 \\
Thiès & 3 & 2,8 \\
Diourbel & 5 & 4,6 \\
Louga & 6 & 5,5 \\
Kaolack & 2 & 1,8 \\
Kaffrine & 1 & 0,9 \\
Matam & 3 & 2,8 \\
St-Louis & 1 & 0,9 \\
Ziguinchor & 1 & 0,9 \\
Foundioungne & 2 & 1,8 \\
Gambia & 1 & 0,9 \\
Mauritania & 1 & 0,9 \\
Overall & 109 & 100 \\
\hline
\end{tabular}




\subsection{Risk Factors and Etiologies of Stroke}

$57.8 \%$ had an ischemic stroke and $42.2 \%$ a hemorrhagic stroke.

\subsubsection{Ischemic Stroke}

Ischemic stroke was found in 63 patients $(57.8 \%)$ with an average age of 39 years.

\section{i. Cardiovascular Risk Factors}

After a clinical and paraclinical analysis of the data collected in our patients, the various cardiovascular risk factors are recorded in the following table.

Table 3. Cardiovascular Risk Factors in ischemic stroke.

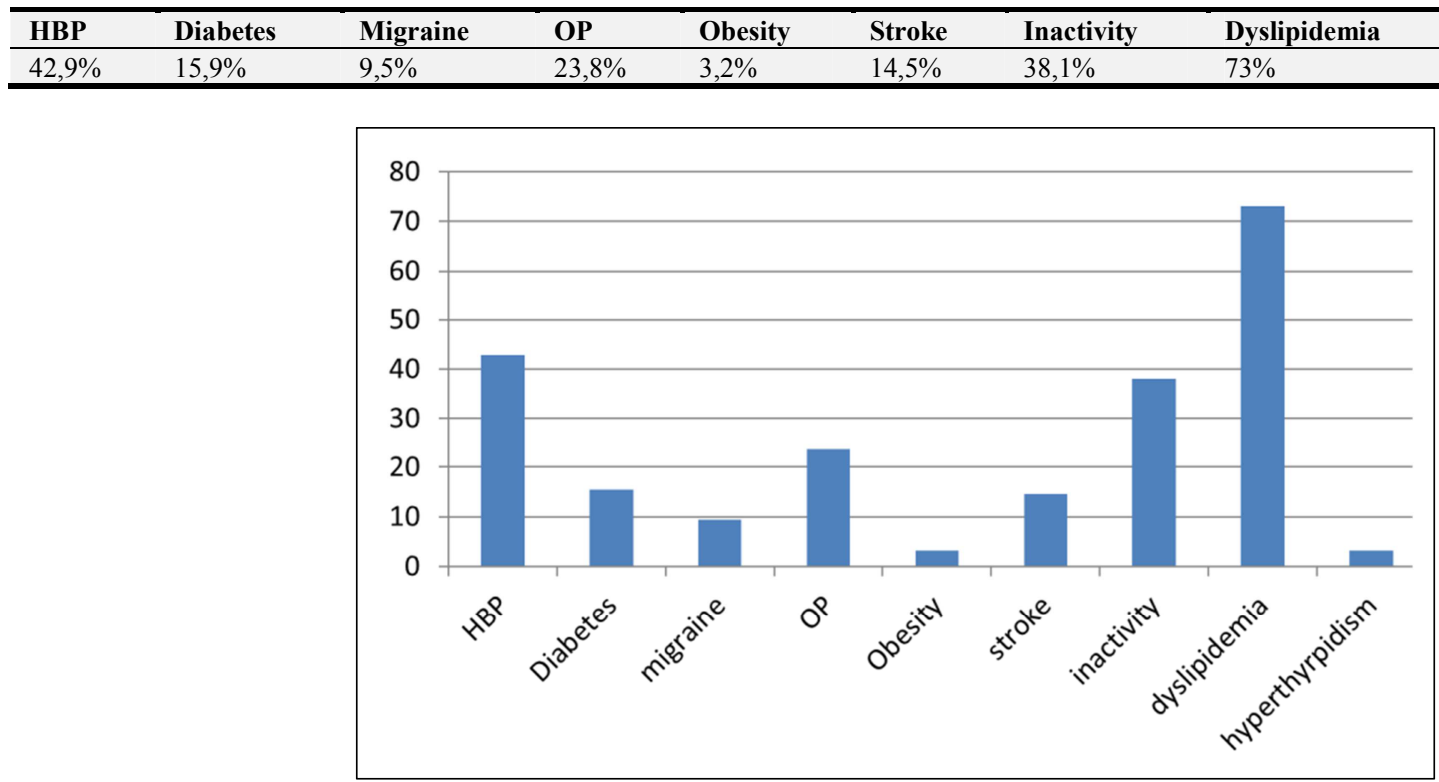

Figure 3. Cardiovascular Risk Factors in ischemic stroke.

HBP: High Blood Pressure

OP: estrogen- progestin pill

\section{ii. Etiologies of Ischemic Stroke}

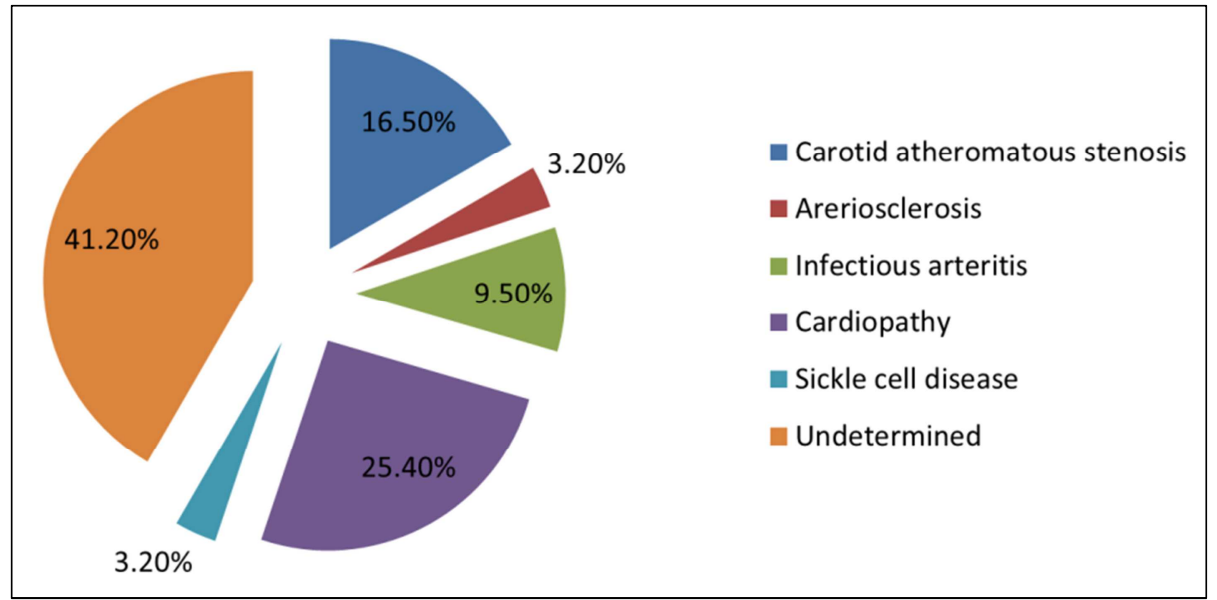

Figure4. Aetiologies of ischemic stroke.

Carotid atheromatous stenosis: 11 patients $(17.5 \%)$

Arteriosclerosis: 2 patients $(3.2 \%)$

Infectious arteritis:6 patients (9.5\%) whose $3(4.8 \%)$ had HIV, $2(3.2 \%)$ syphilis, and 1 post meningitis arteritis $(1.6 \%)$.

Heart disease: $16(25.4 \%)$ whose $2(3.2 \%)$ myocardial infarction; $2(3.2 \%)$ enlarged hypertrophic cardiomyopathy; 1 (1.6\%) ischemic cardiomyopathy; 2 (3.2\%) complete arrhythmia by atrial fibrillation; 3 (4.8\%) mitral insufficiency; $2(3.2 \%)$ mitral stenosis et $3(4.8 \%)$ mitral disease; 1 (1.6\%) aneurysm of the interatrial septum.

Sickle cell disease: 2 patients $(3.2 \%)$

Undetermined: $41.2 \%$. 
Table 4. TOAST classification.

\begin{tabular}{ll}
\hline Embolic heart disease & $25.4 \%$, \\
Macroangiopathy & $17.5 \%$, \\
Autres & $15.9 \%$ \\
Microangiopathy & $7.94 \%$, \\
Undetermined & $41.2 \%$. \\
\hline
\end{tabular}

\subsubsection{Hemorrhagic Stroke}

46 patients or $42.2 \%$ had a hemorrhagic stroke with an average age of 40 years.

\section{i. Cardiovascular Risk Factors}

The cardiovascular risk factors found are shown in table $\mathrm{V}$

Table 5. Cardiovascular risk factors of hemorrhagic stroke.

\begin{tabular}{|c|c|c|c|c|c|c|c|c|}
\hline НВР & Diabetes & Migraine & OP & Obesity & stroke & Inactivity & Tobacco & Dyslipidemia \\
\hline $80.4 \%$ & $4.3 \%$ & $23.9 \%$ & $6.5 \%$ & $2.2 \%$ & $11.1 \%$ & $59.1 \%$ & $2.3 \%$ & $58.7 \%$ \\
\hline
\end{tabular}

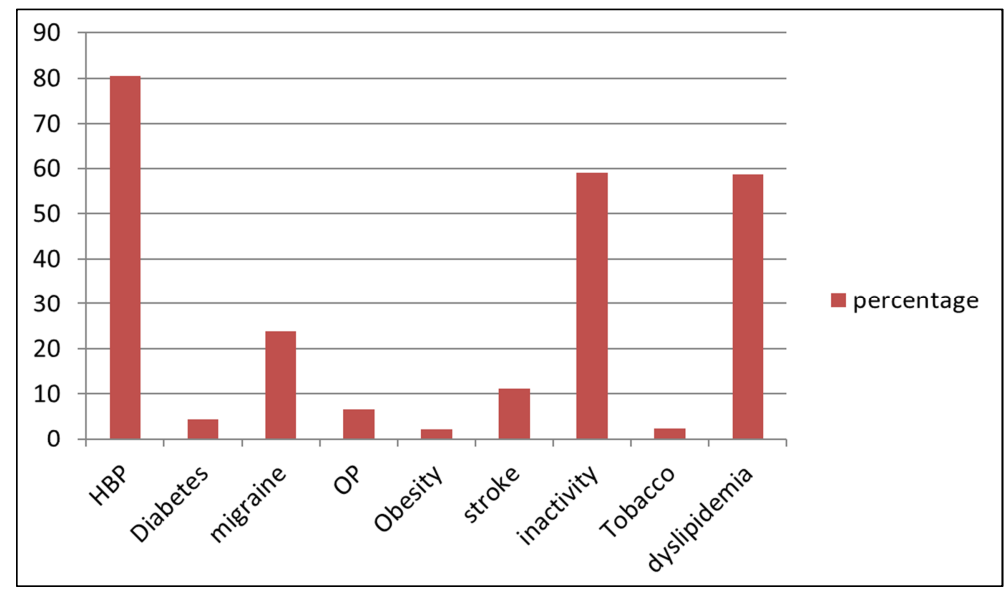

Figure 5. Cardiovascular risk factors of hemorrhagic stroke.

\section{ii. Etiologies of Hemorrhagic Stroke}

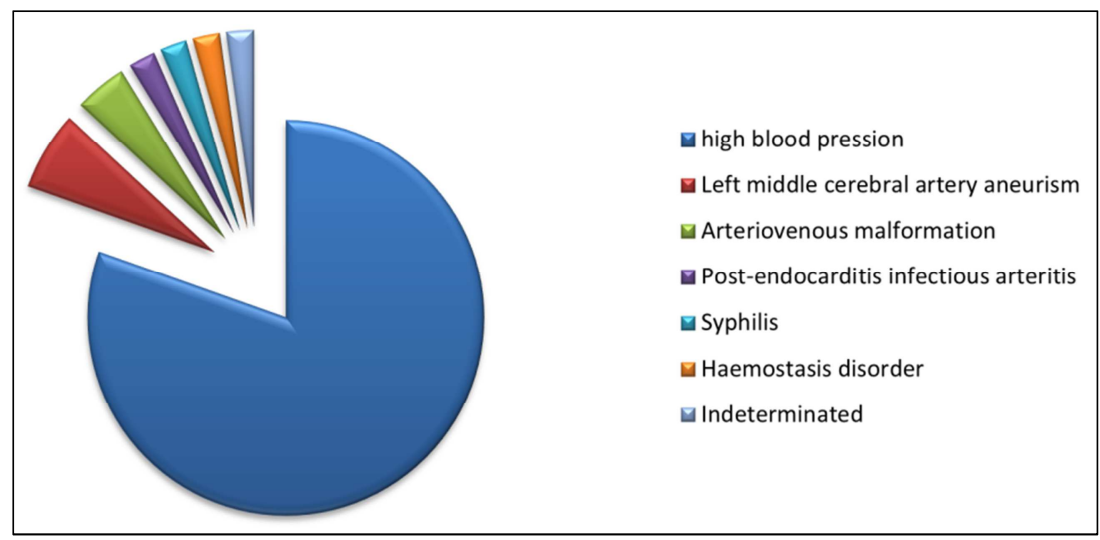

Figure 6. Etiology of hemorrhagic stroke.

High blood pressure (HBP):37 cases (80.4\%)

Left middle cerebral artery aneurism: 3 cases $(6.5 \%)$

Arteriovenous malformation: 2 cases $(4.3 \%)$

Post-endocarditic infectious arteritis: 1 case $(2.2 \%)$

Syphilis: 1 case $(2.2 \%)$

Hemostasis disorder: 1 case $(2.2 \%)$

Undetermined: 1 case $(2.2 \%)$

\section{Discussion}

\subsection{Epidemiological Data}

In our study, 109 patients aged 17 to 50 years were recruited over a period of twenty-two months. Indeed the incidence is low; only 12 to $15 \%$ of strokes occur in under 50 $[12 ; 24]$ according to the available data. According to Sturm [24], the incidence rate varies between 10 and 23 cases per 100,000 inhabitants worldwide. In the study by Betsy B. Love [16] the incidence rate was 9 and 7 per 100,000 
inhabitants for ischemic stroke and hemorrhagic stroke respectively.

According to the Northern Manhattan Study (NOMAS), the age adjusted stroke incidence rates in young adults were then calculated in blacks, whites and Hispanics. The incidence of all strokes in blacks was 25 per 100,000 per year; in Hispanics, 26; and in whites, 10. These rates corresponded to an increased age adjusted relative risk of all stroke of 2.4 (95\% CI: $0.8-6.7)$ in blacks compared with whites and $2.5(1.1-5.8)$ in Hispanics compared with whites. For ischemic stroke, the incidence rates were 11 per 100,000 per year in Blacks, 11 in Hispanics, and 7 in whites [28].

However, it is important to recognize the recrudescence of stroke in young people, as indicated by a study of the health monitoring institute in France, the stroke rate increased by more than $10 \%$ in those under 50 between 2002 and 2008, a study of the population of Kentucky (USA) shows that in 10 years the proportion of stroke has increased from 12.9 to $18.6 \%$ among people aged between 20 and 54 [27].

The most representative age group in our sample is between 36 and 45 years old. This fits well with the literature data [18]. The risk increases with age and the likelihood of being a victim of stroke doubles for every 10 years. [12] According to Sturm [24], only $30 \%$ of strokes in young people occur in people under 35 years of age.

The average age in our series was 39.95 years old. This is relatively close to that of Balogou which was 38.5 years old [3]. In another study, the average age was smaller: 33 years for the study of Mohamed Chraa [6].

\subsection{Different Types of Stroke}

Our study presented a rate of $57.8 \%$ of ischemic stroke and $42.2 \%$ of hemorrhagic stroke. These results do not match with the literature data which were of $80 \%$ for ischemic stroke and $20 \%$ for hemorrhagic stroke [15]. Sène Diouf et al (in 2006 and 2008) in Senegal reported respectively 35.3\% of hemorrhagic stroke vs. $64.7 \%$ of ischemic stroke and $48.6 \%$ of hemorrhagic stroke vs. $51.4 \%$ of ischemic stroke. In Mauritania, studies found $48 \%$ of hemorrhagic stroke vs. $52 \%$ of ischemic stroke [1]. Balogou et al found in Lomé in young subjects $37.6 \%$ of hemorrhagic stroke vs $62.4 \%$ ischemic stroke [3].

The study of Sturm et al [24], done in Aquila, Italy, reported a result close to ours with $43 \%$ hemorrhagic stroke and $57 \%$ ischemic stroke in the under 50 years old. According to Ovbiagele et al [20] the hemorrhagic stroke rate in black Americans was 20 to $25 \%$ of all stroke, $25 \%$ in Asians and $17 \%$ in Westerners. Singhal [22] found that in the under 50 years old in USA, 50\% were hemorrhagic stroke and $50 \%$ ischemic. In total we notice that on average the rate of ischemic stroke in the young subject varies between 50 and $60 \%$ vs 40 to $50 \%$ for hemorrhagic stroke $[24 ; 22 ; 20]$.

\subsection{Risk Factors}

Oestroprogestative contraception intake

Eighteen patients $(16.5 \%)$ used oestroprogestative contraception. However, in our study, only 7 (or 6.4\%) patients had a stroke with no other cardiovascular risk factor found than the use of oestroprogestative pills, which is close to Griffiths' et al data where the use of oestroprogestative contraception was related with $8 \%$ of strokes [11]. The first publication on a possible association between oral contraception and stroke in 1962 is followed by several other studies that have shown an estimated risk between 3 and $4 \%$ for ischemic stroke and $1 \%$ for hemorrhagic stroke. This is in agreement with our data since 15 patients under oestroprogestative had an ischemic stroke or a rate of $13.8 \%$ while only 3 patients or $2.7 \%$ under oestroprogestative had a hemorrhagic stroke.

According to SZTAJZEL [25] the majority of the studies do not show an increase in the risk of hemorrhagic stroke in people under oestroprogestative pills in the industrialized countries; but in developing countries the risk increases with age beyond age 35 and with other cardiovascular risk factors such as hypertension and tobacco. According to the same author, the risk of stroke related to oral contraception is low. On a ratio of 1 woman in 200,000 / year, there must be 5880 women who take contraception during one year to train a single stroke. However, this risk is multiplied by 5 if the patient has other cardiovascular risk factors and is over 35 years old. This is the case of 11 patients on the 18 of our series who were under oestroprogestative pills and presenting other risk factors such as: hypertension, hypercholesterolemia, a history of stroke, diabetes. According to F. Woimant [26] the risk associated with oral contraception is lower with the last generation and microdose pills $(\mathrm{OR}=4.53$ if $>50 \mu \mathrm{g}$ estrogen and 2.08 if $<50 \mu \mathrm{g}$ estrogen). In patients with a cardiovascular risk factor, it is recommended to use non-hormonal contraception or an intrauterine device.

\section{Postpartum and pregnancy}

Among our patients, only 7 cases (i.e. 6.4\%) were postpartum, including 4 cases of ischemic stroke and 3 hemorrhagic strokes, only one woman $(0.9 \%)$ was pregnant with toxemia of pregnancy. In the Chraa study $3.8 \%$ of the cases were postpartum and none in pregnancy.

According to Woimant [26] the risk of stroke is not really increased during pregnancy. On the other hand, during the postpartum period, the risk is multiplied by 12 and is related to the gestational hypertension, the maternal age and the achievement of a caesarean section. Our 7 patients all had a pregnancy-related hypertension and one of them also had eclampsia.

According to Ferro et al [9] the occurrence of stroke in pregnancy is rare. During the gravid-puerperal period, some abnormalities may explain the risk of stroke; these are cardiomyopathy of the per partum, a blood hypercoagulability or an alteration of the vascular walls. But a stroke occurring during pregnancy is not a contraindication for a subsequent pregnancy, the risk of recurrence being low [9].

According to Khan and Wasay [14], the causes of stroke in the gravid-puerperium found in their study were: cerebral 
venous thrombosis $(49.1 \%)$, emboligenic heart disease $(10.9 \%)$, macroangiopathy $(10 \%)$, microangiopathy $(3.6 \%)$, vasculitis $(2.7 \%)$, carotid dissection $(9 \%)$, and hypercoagulability (3.6\%). In our series, of the 4 patients with early ischemic stroke in postpartum, only one had hypertrophic dilated cardiomyopathy as etiology. In the other 3 , no etiology was found, except for arterial hypertension as a risk factor.

The risk of ischemic stroke or intracerebral hemorrhage during pregnancy and the first 6 weeks postpartum was 2.4 times greater than for nonpregnant women of similar age and race, according to the Baltimore-Washington Cooperative Young Stroke Study. The excess risk of stroke (all types except subarachnoid hemorrhage) attributable to the combined pregnancy/postpregnancy period was 8.1 per 100 000 pregnancies. [28].

\section{Migraine}

In our study, 11 patients $(15.6 \%)$ were migraine patients compared to $3.8 \%$ in the Chraa series [6]. According to Love et al [16] migraine is involved in 10 to $15 \%$ of strokes. The literature indicates that migraine doubles the risk of stroke [17]. According to Sturm et al [24] the risk is 2.36\%; this risk triple in case of association with tobacco and quadruple in case of oestroprogestative pills intake. According to Griffiths et al [11], 34 to $35 \%$ of migraine patients who smoke and who take oestroprogestative pills have a stroke.

Tobacco

A study done in Baltimore - Washington aims a strong dose - response relationship between smoking and the risk of ischemic stroke in young women and reinforces the need for aggressive smoking cessation efforts among young adults. The odd ratio comparing current smokers to never smokers was $2.6(\mathrm{p}<0.0001)[10]$.

Only one of our patients was smoker $(0.9 \%)$. In the Azankpan study [2] in Benin only $0.26 \%$ of women were smokers. This could be explained by the low prevalence of female smoking in our countries. Tobacco reduces vessel compliance, promotes platelet aggregation, lowers HDL cholesterol, and promotes hemoconcentration. The relative risk is 1.5 , this risk is 7 times higher in women combining smoking and oestroprogestative pills use [20]. The combination oestroprogestative-tobacco-migraine is an explosive cocktail multiplying by 30 the risk of stroke [24].

\subsection{Etiologies of Ischemic Strokes}

We found approximately the same rate of emboligenic heart disease as Bellalem et al [4]; 20.5\% for Bellalem and $25.4 \%$ in our sample. This is consistent with the literature: The main etiology of cerebral ischemia in young people in industrialized countries is represented by cervical arterial dissections and in developing countries by emboligenic heart disease, particularly rheumatic heart disease [15]. Microangiopathies for Bellalem et al were $15.8 \%$ vs 9.74 in our series; $13.7 \%$ atherosclerosis of large arterial trunks or macroangiopathy vs $17.5 \%$ in our study; others at $9.8 \%$ and $40.2 \%$ of undetermined causes' vs. $41.2 \%$ in our series. The high rate of carotid atherosclerosis in our study can be explained by the high proportion of dyslipidemia at $73 \%$ in our risk factors.

Diagne [8] found $4.5 \%$ atherosclerosis significantly lower than ours $(17.5 \%)$. With a cardiac disease rate $54.16 \%$ higher than ours; this can be explained by the fact that the collection of patients was carried out in addition to the neurology department of Fann, the cardiology department of the University Hospital A. Dantec. The other causes were in $12.5 \%$ and $16.5 \%$ of indeterminate causes. In its other causes there were arteritis with HIV1 and another arteritis of unspecified cause. In our sample, we found 3 cases of HIV-1 arteritis (4.8\%) and $7.4 \%$ of HIV in the Onwuchekwa et al [19] study in Nigeria, this may be due to the HIV endemic in sub-Saharan Africa. HIV infection increases the relative risk of infarction $(\mathrm{RR}=9.1 \%)$ and bleeding $(\mathrm{RR}=12.7 \%)[1]$.

Chraa [6] in Morocco found 33.5\% of heart disease with a predominance of rheumatic heart disease vs $26 \%$ in the DeSylva et al [7] study in Sri Lanka; $11.7 \%$ atherosclerosis vs $17.5 \%$ in our study; $40.6 \%$ undetermined cause and other causes are $11 \%$ syphilitic arteritis vs $3.2 \%$ in our series; $2.3 \%$ cervical arterial dissections; $1.6 \%$ of protein deficit $\mathrm{C}$ and $\mathrm{S}$ and $0.8 \%$ of antiphospholipid syndrome vs $8 \%$ in a study in Sri Lanka [7]. The last three causes which are carotid dissection, protein $\mathrm{C}$ and $\mathrm{S}$ deficiency and antiphospholipid syndrome were not found in our patients.

Onwuchekwa [19] et al in Nigeria found $13 \%$ of emboligenic heart diseases vs $25.4 \%$ in our study; $7.4 \%$ HIV infection; $3.7 \%$ of sickle cell disease SS and 1 case of ovarian cancer (i.e. $1.9 \%$ ).

In our study the rate of sickle cell disease SS 3.2\%, is close to that of Onwuchekwa [63]: 3.7\%. Balogou [3] found a lower rate of $1.4 \%$. Studies have shown that the incidence of stroke in sickle cell SS increases with age: 310/100000 people per year for under 18 years, $360 / 100000$ people per year between 18 and 34 years, 1160/100000 people per year between the ages of 34 and 65 , and $4700 / 100000$ people per year for those over 65 [23]. This may justify the low prevalence of stroke in sickle cell disease, found in our study.

\subsection{Etiologies of Hemorrhagic Stroke}

High blood pressure: 37 cases $(80.4 \%)$, is the main cause in our series. This rate of $80.4 \%$ reflects the high prevalence of hypertension in our country even among young people. In a study done in urban population in Saint-Louis of Senegal [21], 13\% of hypertension is found between 15 and 24 years; $23 \%$ of HTA between 25 and 34 years; 33\% HTA between 35 and 44 years and $58 \%$ for those aged 45 to 54 years. This very high rate of hypertension at $80.4 \%$ is in agreement with the studies of Balogou [3] in Togo and Kalita [13] in India, which found respectively an HTA rate of $79.9 \%$ and $79 \%$. In the Black American study by Love B. [16], there is a lower rate of hypertension $(64.2 \%)$. According to another study by Sturm [24] in the United States, in the general population the rate of hemorrhagic stroke due to hypertension was only $13 \%$. The question is to know if race and environmental factors are involved in the occurrence of hypertension.

Vascular malformations were $10.8 \%$ in our study, Love B 
[16]; et al found $4.5 \%$ aneurysm and $4.5 \%$ arteriovenous malformation. Kalita [13] et al had $4.2 \%$ vascular malformation in their series, whereas Sturm [24] found 50\% in their sample. In the literature [5] vascular malformations account for only 5 to $10 \%$ of the causes of hemorrhagic stroke in the general population but at least a third of the cases in the young subject.

2 cases of infectious arteritis (4.4\%) were reported vs. $0.5 \%$ in the Kalita study [13].

Disturbances in haemostasis were found in one patient (2.2\%); while Kalita [13] had found 4\% in his study; Love B. [16] found in her sample $3 \%$ of hemostasis disorders due to the use of anticoagulants.

However, the determination of factors VIII and IX in search of hemophilia as well as the search for thrombocytopathy had not been carried out for lack of means. In addition, Kalita found cerebral thrombophlebitis in $2.2 \%$ of patients. Undetermined causes affected 1 of patients (2.2\%) while Love B. found $22.4 \%$ and Kalita 9.2\%. Other causes to know: Tumor, cavernoma, cerebral thrombophlebitis, were not found in the study.

\section{Conclusion}

Stroke is a serious pathology that can cause serious sequels. It should be prevented by the management of cardiovascular risk factors and etiologies. Estrogen-progestin intake is not a major risk factor for stroke unless it is associated with other cardiovascular risk factors such as hypertension, diabetes, obesity and tobacco. The cardiovascular risk factors should therefore be screened and treated in the young woman to reduce the occurrence of stroke.

\section{References}

[1] Adoukonou TA, Vallat JM, Joubert J. et al. Prise en charge des accidents vasculairescérébraux en AfriqueSubsaharienne. Revue neurol2010; 16: 882-93.

[2] AZANKPAN E. R. Prévalence des facteurs de risque des AVC en population générale à Cotonou (Bénin). Thèse de doctorat en médecine: UCAD 2010. $\mathrm{N}^{\circ} 58$.

[3] BALOGOU A. A. K., GRUNITZKY E. K., ASSOGBA K. et al. Strokes among youth (15 to 45 years old) in the neurological department of the medical teaching hospital, campus Lomé. AJNS 2008; 27: 1-2.

[4] Bellalem A, Amroune AA, Amiri L et al. Epidémiologie des accidents vasculaires cérébraux ischémiques et classification des sous types selon les critères TOAST à Sétif en Algérie. Revue neurol2007; 18: 9-11.

[5] CAEN LYON O. Accident vasculaire cérébral. Neurologie, Médecine sciences, Flammarion France 2009; 37: 84-196.

[6] Chraa M, Louhab N, Kissani N. Stroke in youngadults: about 128 cases. Pan Africanmedical journal 2014; 17: 1-7.

[7] DE Sylva. R, Ranjani Gamage, Wewelwala C et al. Young strokes in Sri Lanka: An unsolvedproblem. J Stroke CerebrovascDis. 2009; 18: 304-308.

[8] DIAGNE N. S. Etiologies des AVC du sujetjeune (à propos de 40 cas). Thèsemédecine: UCAD $2010 . \mathrm{N}^{\circ} 66$.

[9] FERRO M. F., MASSARO A. R., Mas J. L. et al. Aetiological diagnosis of ischaemic stroke in youngadults. Neurology 2010; 9: 1085-1096.

[10] Bhat Viveca M., John W. Cole, John D. Sorkin and al. Dose Response relationship between cigarette smoking and risk of ischemic stroke in young women. Stroke 2008; 39: 24392443.

[11] GRIFFITHS. D., STURM J et al. Epidemiology and Etiology of Young Stroke. Stroke Research and Treatment 2011, Article ID 209370, 9 pages.

[12] Horde P. Accident vasculairecérébral- statistiques (France). Santé medecine.net 2014; tous droits réservés: 1-3.

[13] Jayantee K., Gourav G., Kumar P. and al. Intracerebral hemorrhage in young from in tertiary neurology center in North India. Journal of neurological sciences 2014; 336: 4247.

[14] KHAN M., WASAY M., MENON B. et al. Pregnancy and puerperium- related stroke in Asian women. Journal of Stroke and CerebrovascularDiseases2013; 22: 1393-1398.

[15] LEYS D., DEFEBVRE L. Pathologie vasculaire cérébrale. Neurologie, Ellipses France 2004; 14: 141-148.

[16] Love B. B., Biller J. Stroke in children and young adults: overview, risk factor, and prognosis. National Vital Statistics Reports Hyattsville, MD 2008; 56: 1-14.

[17] MARQUIS I. Les femmes et les AVC: Risques spécifiques, pronostics défavorables. Fondation des maladies du cœur du Québec.

[18] MASSARDIER E. G. Accidents ischémiques cérébraux (AIC) du sujet jeune, généralités. Stoke 2009; 40: 1195-1203.

[19] Onwuchekwa A. C, Onwuchekwa R. C, Asekomeh E. G et al. Stroke in young Nigerian adults. Journal of vascular nursing, 2009; 27: 98- 102.

[20] OVBIAGELE B., NGUYEN-HUYNH. Stroke Epidemiology: Advancing Our Understanding of Disease Mechanism and Therapy. Neurotherapeutics2011; 8: 319-329.

[21] Pessinaba S, Mbaye A, Yabeta GA, Kane A, Ndao CT, NdiayeMBet al. Prévalence et déterminants de l'hypertension artérielle et les facteurs de risque associés: des données à partir d'une enquête transversale basée sur la population de Saint Louis, Sénégal. Cardiovascular Journal Afrique 2013; 24: $180-3$.

[22] SINGHAL B. A., BILLER J., ELKIND M. S. et al. Recognition and management of stroke in young adults and adolescents. Neurology2013; 81: 1089-1097.

[23] STROUSE J. J., LORI C., LANZKRON S. et al. The Excess Burden of Stroke in Hospitalized Adults with Sickle Cell Disease. Am J Hematol, 2009; 84: 548-552.

[24] Sturm JW, Mackay MT, Thrift AG. Stoke among women, ethnic groups, young adults, and children. Handbook of Clinical Neurology 2009; 92: 337-53. 
[25] SZTAJZEL R. et DEVRUJST G. Sujet Neurologie. Revue médicale suisse; 2390: 1-4.

[26] WOIMANT F. Particularité de l'accident vasculaire cérébral de la femme. Réalitéscardiologiques2012; 290: 30-34.

[27] WOIMANT F; plus d'AVC chez le jeune. Medscape France 2012; 1: 1-5.
[28] Donald Lloyd-Jones, MD, ScM, FAHA; Robert J. Adams, MD, FAHA; Todd M. Brown, MD; and al. Heart Disease and Stroke Statistics-2010 Update. Circulation AHA 2010. E46260.

[29] Emelia J. Benjamin, Michael J. Blaha, Stephanie E. Chiuve, Mary Cushman, Sandeep R. Das, heart disease and stroke statistics - 2017 update: a report from the American heart association. Circulation AHA 2017; 135:e146-e603. 